\title{
Soins gérés/assurance de responsabilité civile - Une analyse objective
}

\section{PAR KIRSTEN NORTH, OD, PRÉSIDENT DÉSIGNÉ DE L'ACO}

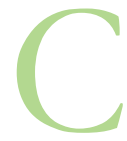

ompte tenu de la désinscription de services optométriques, le rôle d'assureur de responsabilité civile prend de plus en plus d'importance et la tendance se poursuivra sans doute. Les sociétés de soins gérés / responsabilité civile considèrent le Canada comme un marché attrayant et lucratif maintenant que sensiblement la moitié de la population du Canada (surtout les personnes en âge de travailler) n'est plus inscrite à l'assurance maladie provinciale.

Libérés des contraintes des programmes d'assurance maladie provinciaux, les membres de l'ACO devraient prendre conscience de l'arme à double tranchant que représentent les assureurs de responsabilité civile. En général, les sociétés à but lucratif sont sans doute plus difficiles d'approche, et imposent des honoraires moins élevés, que ne l'ont jamais été les régimes d'assurance des provinces.

Des fournisseurs de soins sous gestion aux États-Unis ont communiqué avec l'ACO pour se renseigner sur le marché canadien. Certaines de ces sociétés estiment être en mesure de faire des affaires avec des filiales canadiennes de clients américains. Ces sociétés peuvent réaliser des économies de coût très importantes en étendant à un autre territoire un programme déjà établi plutôt que de partir de zéro.

Même si beaucoup d'assureurs américains ont une forte présence nationale, leur représentation dans les États individuels n'est pas uniforme aux États-Unis. La solidarité est essentielle au succès que ces secteurs de compétence ont obtenu.

Il est critique de souligner aux membres qu'une offre trop belle pour être vraie l'est sans doute. Il peut sembler au départ attrayant de diminuer les honoraires professionnels en échange d'une augmentation possible du volume de patients, mais cela pourrait avoir un effet négatif important sur le résultat final. Le praticien pourrait aussi perdre son pouvoir de décision clinique tout en demeurant pleinement responsable des décisions qui sont prises.

Voici quelques scénarios susceptibles d'intéresser les praticiens :

- Les patients sont typiquement dirigés vers un cabinet optométrique au moyen de listes de «fournisseurs privilégiés ».

- La plupart des fournisseurs de soins gérés établissent les honoraires des examens sans que le praticien ait la possibilité « d'équilibrer la facture » pour toucher ses honoraires habituels.

- Des procédures à suivre pour chaque client risquent d'être imposées, supprimant ainsi le jugement clinique (mais non la responsabilité) de l'équation.

- Il n'y a aucune option pour « limiter » le nombre de «patients en gestion des soins » examinés.

- Les produits ophtalmiques utilisés, et leur prix, risquent aussi d'être imposés.

- Un comité interne « d'assurance de la qualité » pourrait examiner les dossiers pour s'assurer que toutes les procédures exigées ont été accomplies - une retenue pécuniaire risque de s'appliquer si des «lacunes » ressortent.

- Les contrats sont typiquement d'une durée de deux à trois ans et leur reconduction, face à une concurrence, risque de faire diminuer les honoraires.

- Si un employé décide d'aller vers un praticien non affilié, ses avantages risquent d'être substantiellement amputés.

Il est de plus en plus apparent que ces modalités ont principalement pour but de rendre le fournisseur de soins gérés plus concurrentiel et d'accroître sa part du marché 1) en réduisant ou en restreignant les services aux patients et 2) en réduisant les honoraires versés aux fournisseurs (optométristes).

Nous incitons fortement les membres de l'ACO à analyser attentivement les réalités des soins gérés. 